\title{
MEASUREMENTS OF IMPEDANCE AND BEAM INSTABILITIES AT THE AUSTRALIAN SYNCHROTRON
}

\author{
Rohan Dowd, Mark Boland, Gregory LeBlanc, Martin Spencer, Eugene Tan (ASP, Clayton, Victoria) \\ John Byrd, Fernando Sannibale (LBNL, Berkeley, California)
}

\section{Abstract}

In this paper we present the first measurements of machine impedance and observed beam instabilities at the Australian Synchrotron. Impedance measurements are made by studying the single bunch behaviour with beam current, using optical and X-ray diagnostic beamlines. An observed coupled-bunch instability, its cause and cure is also discussed.

\section{OVERVIEW}

The Australian Synchrotron is a newly operating 3rd generation light source facility located in Melbourne, Australia. The $3 \mathrm{GeV}$ storage ring is 216 metres in circumference and can store a beam of up to $200 \mathrm{~mA}$ current. A design overview can be found in [1].

\section{BEAM DIAGNOSTICS}

There are two diagnostic beamlines at the Australian Synchrotron, an X-ray and an optical beamline. Details of the diagnostics beamlines can be found in [2].

The X-ray diagnostic beamline uses the X-ray light from a bending magnet and passes it through a pinhole array. The multiple pinhole images are then projected onto a fluorescent screen and imaged by a CCD camera. The camera optics allow for a $10 \mu \mathrm{m}$ resolution of the transverse beam size.

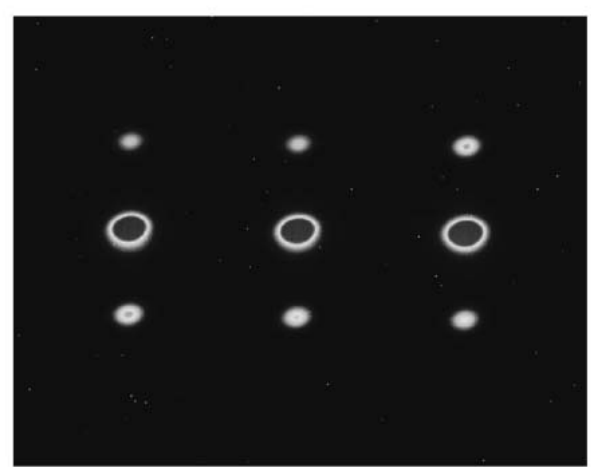

Figure 1: Typical image from the X-ray Diagnositc beamline

The Optical Diagnostic beamline uses the light from a dipole magnet after it has been passes through a optical chicane. This light is then split and analysed by several different devices, given information about bunch length, beam 05 Beam Dynamics and Electromagnetic Fields emittance and even the fill pattern. Most important for this analysis is the fast sweeping streak camera that allows us to determine single bunch length, with a picosecond resolution.

\section{SINGLE BUNCH STUDIES}

For single bunch studies we injected up to $10 \mathrm{~mA}$ into a single bucket of the storage ring. As high amounts of RF power was observed from kicker pickups during single bunch operation, $10 \mathrm{~mA}$ was chosen as an upper current limit in these studies. This minimised potentially harmful effects of reflected RF power in our spectrum analyser and kicker amplifiers.

\section{Synchronous Phase advance}

The energy lost from a bunch due to the wakefields generated by it can be characterised by its loss factor, $k_{l}$. The loss factor is generated by resistive impedances in the machine usually expressed in units of $\mathrm{V} / \mathrm{pC}$, so the energy lost by the beam is then $\Delta E=-k_{l} \cdot q^{2}$, with $q$ being the bunch charge.

For the beam to remain circulating, the energy lost must be made up and the bunch will move up the phase of the RF cavities in order to gain more energy per turn. The change in synchronous phase of the bunch will give you the energy loss due to the impedance of the machine.

To observe the synchronous phase advance, we used the streak camera on the optical diagnostic beamline. To allow us to see small phase shifts, which manifest as time shifts, a reference phase is needed. We filled six buckets in the ring with small amounts of current $(0.3 \mathrm{~mA})$ to act as a reference, and then one bucket was filled from 0.5 to $9 \mathrm{~mA}$. As the test bucket was filled, the bunch was observed to move in time with respect to the reference bunches. The results are shown in figure 2.

The energy loss is $\Delta E=-k_{l} \cdot q^{2}$, therefore $\Delta V=$ $-k_{l} . q=-k_{l} . I_{b} / f_{0}$ Where $I_{b}$ is the bunch current and $f_{0}$ is the revolution frequency. To recover this energy lost an equal and opposite voltage shift in the RF is needed. For a small change this would be $\Delta \hat{V}=V_{0} \cos \left(\phi_{s}\right) \cdot \Delta \phi_{s}$, with $V_{0}$ the peak RF voltage and $\phi_{s}$ the synchronous phase. So equating these we have:

$$
\begin{aligned}
V_{0} \cos \left(\phi_{s}\right) \cdot \Delta \phi_{s} & =k_{l} \frac{I_{b}}{f_{0}} \\
\Delta \phi_{s} & =\frac{k_{l} I_{b}}{V_{0} \cos \left(\phi_{s}\right) f_{0}} \\
\Delta \tau & =\frac{k_{l} I_{b}}{\omega_{R F} V_{0} \cos \left(\phi_{s}\right) f_{0}}
\end{aligned}
$$

D04 Instabilities - Processes, Impedances, Countermeasures 


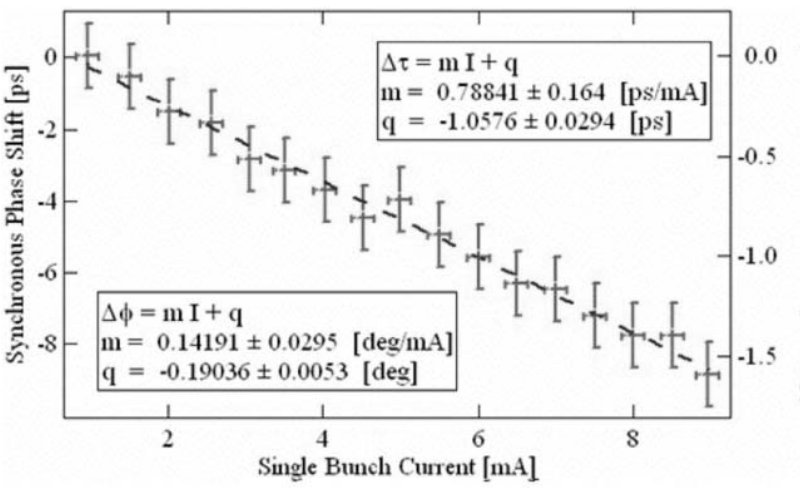

Figure 2: Synchronous phase advance with increasing single bunch current measured at an RF voltage of $2.4 \mathrm{MV}$

In equation 1 we have moved to the time domain, where $\Delta \tau$ is the time shift in the bunch centroid, as seen on the streak camera. Using our obtained result of $\Delta \tau=$ $0.78841 \pm 0.164 \mathrm{ps} / \mathrm{mA}$ we obtain a machine loss factor of $7.48 \pm 1.6 \mathrm{~V} / \mathrm{pC}$. For Gaussian bunches

$$
k_{l}=\frac{R}{2 \sqrt{\pi} \sigma}
$$

giving us a total ring resistance of $R=690 \pm 140 \Omega$.

\section{Potential well Distortion}

As a bunch gains more and more charge, the induced fields from it start to modify the RF potential in which it sits, distorting the shape of the bunch from a pure gaussian. The Hassinski equation [3], describes the equilibrium longitudinal particle distribution within a bunch in the presence of an arbitrary longitudinal wake field. It is given as

$$
\lambda(z)=K \cdot e^{\left(\frac{-z^{2}}{2 \sigma_{0}^{2}}+\frac{\int_{0}^{z} V_{i n d}\left(z^{\prime}\right) d z^{\prime}}{V_{R F} \sigma_{0}^{2}}\right)}
$$

Where $\sigma_{0}$ is the natural bunch length, $V_{R F}^{\prime}=$ $-\left(\omega_{R F} / c\right) . V_{0} \cos \left(\phi_{s}\right)$ is the slope of the RF voltage at synchronous phase, and the induced voltage, $V_{i n d}$, is

$$
V_{\text {ind }}(z)=-\int_{0}^{\infty} W^{\|}\left(z^{\prime}\right) \cdot \lambda\left(z-z^{\prime}\right) d z^{\prime}
$$

This gives a particle distribution that is a Gaussian multiplied by a distortion term that depends on the wakefield generated by the bunch, which in turn depends on the particle distribution. The Hassinski equation is difficult to solve analytically due to this self-consistency condition. However if we express $V_{\text {ind }}$ as a simple linear combination of $\lambda(z)$ and $\lambda^{\prime}(z)$, as would arise from a series combination of fixed resistance $\mathrm{R}$ and fixed inductance $\mathrm{L}$ we get

$$
V_{\text {ind }}(z)=-c q\left[R \lambda(z)+c L \lambda^{\prime}(z)\right]
$$

and the equation becomes solvable via an iterative method. Using this form we can compare the predicted bunch 05 Beam Dynamics and Electromagnetic Fields lengthening with current for a given $\mathrm{R}$ and $\mathrm{L}$ and compare it with measurements. Using $R=690 \Omega$ from above, different values for $L$ were input and compared with the data. The amount of bunch lengthening was found to be quite sensitive to the choice of $\mathrm{L}$. The best fit was found with $\mathrm{L}=22.5 \mathrm{nH}$ and is shown in figure 3 . The divergence of the high current data points from the line may be due to the emergence of single bunch instabilities beginning to have an effect on the bunch length.

Assuming a predominately inductive machine impedance we can extract the machine impedance using

$$
\left(\frac{Z}{n}\right)_{e f f}=\omega_{0} L=0.196 \Omega
$$

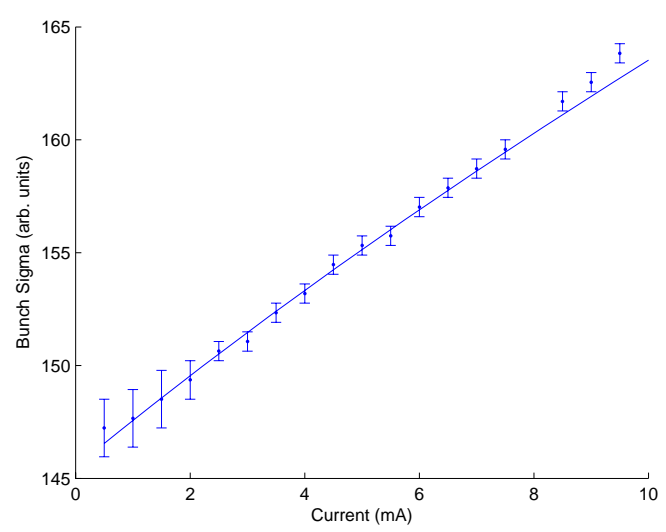

Figure 3: Bunch lengthening predictions for $\mathrm{R}=690 \Omega$ and $\mathrm{L}=22.5 \mathrm{nH}$ plotted against measured values.

\section{Microwave instability}

Another single bunch effect that can give us an estimate of the machine impedance is the microwave instability. This instability manifests when the peak bunch current exceeds a stability limit determined by the machine impedance. When this occurs the bunch turbulently lengthens until the peak current falls below the threshold level. This causes an increase in the energy spread of the beam and can be seen as a sudden increase in the horizontal beam size (and to a lesser extent the vertical beamsize) in a dispersive region of the lattice. Our X-ray diagnostic beamline's source point is in a dispersive region and thus sensitive to this effect.

We analysed images from the X-ray beamline by fitting a Gaussian profile to the $\mathrm{X}$ and $\mathrm{Y}$ distributions of a pinhole array image. Fits were made from 1 to $10 \mathrm{~mA}$ and the sigma of the Gaussian extracted. The results can be seen in figure 4 and show no increase in the beam size up to 10 $\mathrm{mA}$. Due to low light intensity at $1 \mathrm{~mA}$, the fits did not work well and resulted in extremely large sigma values for the first fit. When we determine the instability threshold for the microwave effect using the value of $Z / n=0.2 \Omega$ found

D04 Instabilities - Processes, Impedances, Countermeasures 
previously we obtain a single bunch current threshold of 18 $\mathrm{mA}$, consistent with these results.
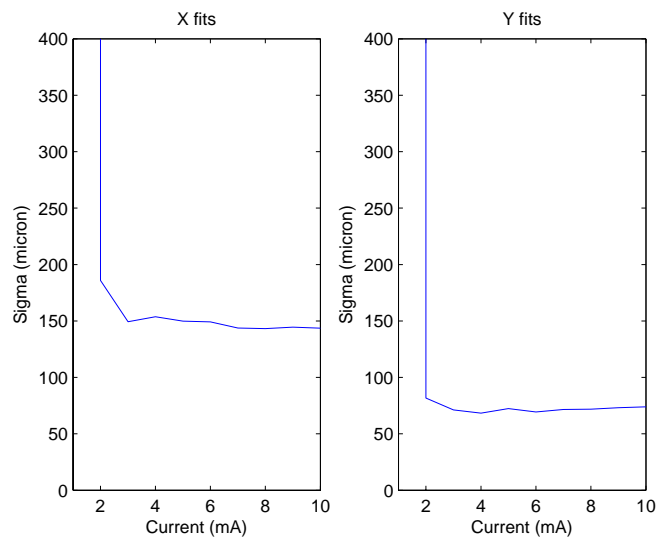

Figure 4: Gaussian fits (top) and plot of sigmas (bottom) in $\mathrm{X}$ and $\mathrm{Y}$ for single bunch currents 1 to $10 \mathrm{~mA}$

\section{COUPLED BUNCH INSTABILITIES}

Apart from the Robinson instability, which is cured with the standard detuning of the RF cavities, we have only observed one coupled bunch instability so far. At high stored beam current (in a 300 of 360 bucket fill) we observe a blow-up of the vertical beam size. Looking at the frequency spectrum of the vertical betatron tunes we see a maximum at the first harmonic above the RF harmonic and then a sharp fall off with increasing harmonic number. This is suggestive of the $1 / \sqrt{\omega}$ pattern for a resistive wall effect. If we consider the transverse impedance of our vacuum chamber using [4]

$$
\frac{Z_{y}(k)}{n}=\frac{Z_{0} L(1-i) \delta}{2 \pi b^{3}} F_{1 y}\left(\frac{a}{b}\right)
$$

where $\delta=\sqrt{2 / \omega \mu \sigma}$ is the skin depth, $\mathrm{L}$ is the chamber length, $\mathrm{b}$ is the chamber height $(16 \mathrm{~mm}), \sigma$ is the chamber resistivity (in our case stainless steel $\sigma=1.4 \times$ $10^{6} \Omega^{-1} m^{-1}$ ) and $F_{1 y}$ is a shape factor which equals 0.822 of our chamber. Inputting our machine parameters gives a vertical impedance of

$$
\frac{Z_{y}(k)}{n}=0.94(1-i) \frac{1}{\sqrt{n}} M \Omega m^{-1} .
$$

Using this value for the impedance we can find the growth rate of the vertical resistve wall equation for a beam current of $100 \mathrm{~mA}$ and an average $\beta_{y}$ of $15 \mathrm{~m}$.

$$
\begin{aligned}
\frac{1}{\tau} & =\frac{1}{2} f_{0} \frac{I}{E}<B>Z_{y} \\
\frac{1}{\tau} & =327 \mathrm{~s}^{-1} \\
\tau & =3.0 \mathrm{~ms}
\end{aligned}
$$

The vertical radiation damping time of the ring, obtained from our simulation model, is $4.8 \mathrm{~ms}$. We can therefore 05 Beam Dynamics and Electromagnetic Fields confidently conclude that the instability we are seeing is a resistive wall effect.

We have seen no evidence of any longitudinal instabilities caused by higher order modes (HOMs) trapped in our cavity. This is attributed to the HOM damping techniques we have installed in the cavities. More information on these can be found in [5].

\section{INSTABILITY CURES, PRESENT AND FUTURE}

The Australian Synchrotron storage ring does not currently have a transverse feedback system, so our only way to cure this vertical instability is to raise the vertical chromaticity to induce head-tail damping. Currently we use a

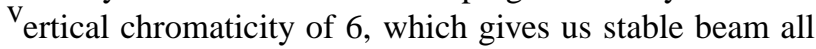
the way up to $200 \mathrm{~mA}$ of stored beam (our design current). This high chromaticity reduces the dynamic apeture considerably, resulting in poor injection efficiency. In order to maintain good injection efficiency, we currently inject with a vertical chromaticity of 3 and then increase the chromaticity after injection to stabilise the beam. In the future we hope to employ a transverse feedback system to damp this instability and allow us to operate at lower chromaticity.

\section{CONCLUSION}

Using Single bunch studies we have obtained an estimate for the total machine impedance of the Australian Synchrotron storage ring. Our analysis gives a total machine inductance of $22.5 \mathrm{nH}$ and therefore an effective $\mathrm{Z} / \mathrm{n}$ of $0.2 \Omega$. This result is supported by our inability to see single bunch microwave effects up to $10 \mathrm{~mA}$. Our investigation of coupled bunch instabilities has led us to conclude that we are experiencing a vertical instability from transverse resistive wall impedance. While this can currently be controlled with chromaticity, as more small-gap insertion devices are added to the ring we will certainly see this problem increase. We hope to have a transverse feedback system in place in the near future to address this.

\section{REFERENCES}

[1] J. Bolderman, D. Einfeld, Nucl. Intr. and Meth., A 521, 2004, pg. 306

[2] M. J. Boland et. al., "X-Ray and Optical Diagnostic Beamlines at the Australian Synchrotron Storage Ring”, EPAC'06, June 2006, Edinburgh, UK.

[3] J. Hassinski, Nuovo Cimento B, 18 (1973) 72 (1997) 56.

[4] R. Gluckstern, J. Zeijts,'Coupling impedance of beam pipes of general cross section " B. Zotter, Phys. Rev. E, 47 (1993) 1 (1997) 56.

[5] J. Wantabe et. al., "Design and cold model test of $500 \mathrm{MHz}$ Damped Cavity for ASP storage Ring RF System”, EPAC'06, Edinburgh, June 2006, pg. 3076

D04 Instabilities - Processes, Impedances, Countermeasures 